\title{
Urachal cyst presenting as a pelvic mass
}

\section{KEYWORDS: computed tomography}

A urachal cyst occurs in the urachal remnant between the umbilicus and bladder [1]. It can present as an extraperitoneal mass in the umbilical region. It is characterized by abdominal pain, and fever if infected. It may rupture, leading to peritonitis

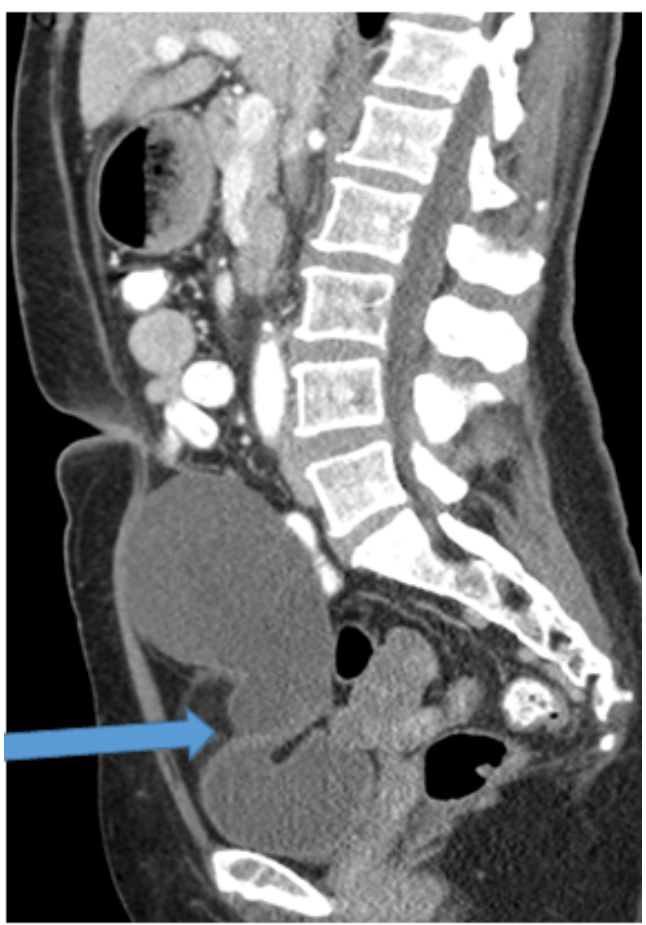

Figure 1. CT scan of abdomen and pelvis; a large fluid density lesion arising from the pelvis. On close observation this cystic lesion is seen arising from the bladder dome and extending towards the umbilicus along the course of urachal remnant. This lesion is consistent with Urachal cyst. or it may drain through the umbilicus. Urachal cysts are usually silent clinically until infection, calculi or adenocarcinoma develops. This patient was asymptomatic who presented with newly discovered pelvic mass (FIGURES 1 and 2).

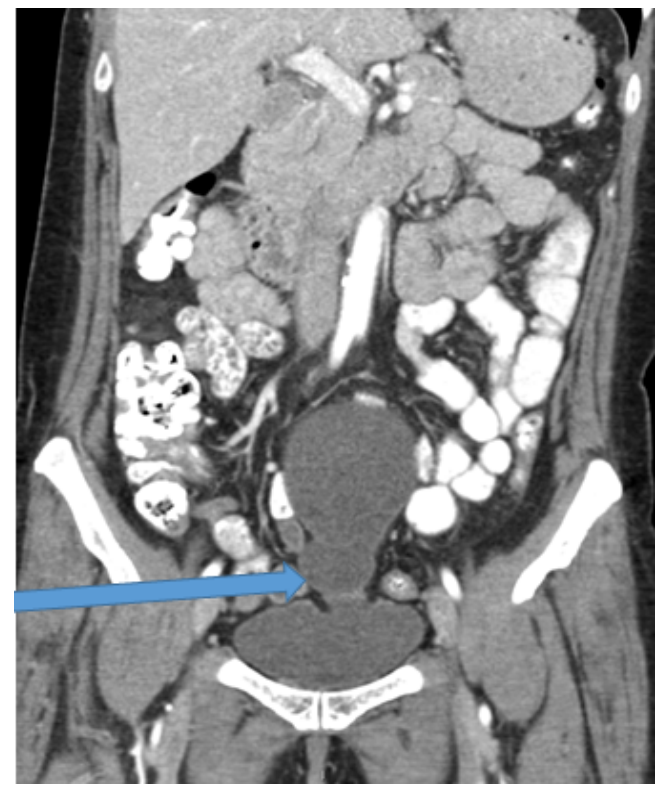

Figure 2. CT scan of abdomen and pelvis; A large $(7 \mathrm{~cm})$ cystic pelvic mass in the midline communicating with the bladder dome.

\section{Sindhu Kumar*}

Department of Radiology, University of Virginia Medical Center, Virginia 22901, USA

*Author for correspondence

\section{REFERENCES}

1. Yu JS, Kim KW, Lee HJ et al. Urachal Remnant Diseases: Spectrum of CT and US Findings. Radiographics. 21, 451-461 (2001). 